\title{
Fine structures of Fe L-emission examined by a new HR-SXES instrument
}

Masami Terauchi ${ }^{1}$, Ryogo Ebisu $^{1}$, Yohei Sato $^{1}$ and Masato Koike ${ }^{2}$

${ }^{1}$ Institute of Multidisciplinary Research for Advanced Materials, Tohoku University, Sendai, Miyagi, Japan, ${ }^{2}$ KPSI, QuBS, QST, Kizugawa, Kyoto, Japan

L-emissions of 3d transition metal elements, which range from 400 to $1000 \mathrm{eV}$, gives us fruitful information about chemical states and/or charged sates of those elements in compounds [1]. L $\alpha, \beta$ emission profile and its intensity reflect the energy distribution and the amount of $3 \mathrm{~d}$ electrons, respectively [2]. The energy position of it is affected by the charged state (chemical shift). The Ll, $\eta$ emission energy, which is due to transitons between inner-shell levels, is changed by a relaxation energy in the final state with a core-hole in $3 \mathrm{~s}$ level [3]. Furthermore, the $\mathrm{Ll}, \eta$ line profile of a $3 \mathrm{~d}$ transition metal element can contain the information of the magnetic moment of $3 \mathrm{~d}$ electrons via $3 \mathrm{~s} 3 \mathrm{~d}$ exchange interaction with the spin of the singly occupied $3 \mathrm{~s}$ level in the final state [2]. Unfortunately, the applied spectrometer did not have an enough energy resolution to analyze the magnetic information in Ll, $\eta$ lines. Thus, a larger energy-dispersion spectrometer designed for TEM [4] was remade as a spectrometer for EPMA [5] and examined L-emission spectra of Fe and Mn.

Figure 1 shows a photo of the spectrometer attached to a EPMA. The distance from a specimen to a valiedline-specing (VLS) grating is the same with that of a commercial spectrometer (right-hand side in Fig.1), but the distance from the VLS grating to a detector is about two times larger than that of the usual one. This means that the two time larger energy-dispersion is expected for this larger spectrometer. Detector is composed of a MCP (7.5 $\square$ m pitch) optically coupled with a CMOS camera.

Figure 2(a) shows Fe-L $\alpha, \beta$ emission spectra of metal-Fe and $\mathrm{Fe} 2 \mathrm{O} 3$. As $\mathrm{L} \alpha, \beta$ emission is due to transitions between $3 \mathrm{~d}$ valence states and $2 \mathrm{p}$ inner-shell levels, the intensity profile reflects bonding states of $3 \mathrm{~d}$ electrons. $\mathrm{L} \alpha$ peak of $\mathrm{Fe} 2 \mathrm{O} 3$ is not symmetric and has a larger width by about $1 \mathrm{eV}$ than that of metal-Fe. It could be due to chemical bonding of $\mathrm{Fe}$ atom with surrounding $\mathrm{O}$ atoms. A small structure between $\mathrm{L} \alpha$ and $\mathrm{L} \beta$ is seen for metal-Fe as indicated by a vertical line but not for Fe2O3. A higher energy position of $\mathrm{L} \alpha$ of $\mathrm{Fe} 2 \mathrm{O} 3$ may be due to a chemical shift of L3(2p3/2) binding energy reflecting the charged state of Fe3+ of Fe2O3. Figure 2(b) shows $\mathrm{Ll}, \eta$ emission peaks of metal-Fe and $\mathrm{Fe} 2 \mathrm{O} 3$. As $\mathrm{Ll}, \eta$ emission is due to transitions between inner-shell levels of $3 \mathrm{~s}$ and $2 \mathrm{p}$, symmetric intensity profile is expected. However, $\mathrm{Ll}$ intensity profile of Fe2O3 shows a shoulder structure in the lower energy side of the peak as indicated by a vertical line unlike that of metal-Fe. This might be due to a $3 \mathrm{~s} 3 \mathrm{~d}$ exchange interaction, an interaction between the magnetic moment of valence $3 \mathrm{~d}$ electrons and the spin of $3 \mathrm{~s}$ level with a hole in the final state. This type of interaction has been reported as a $3 \mathrm{p} 3 \mathrm{~d}$ exchange interaction for $\mathrm{K} \beta$-emission spectra of high spin states of Mn ions [6].

Present high energy-resolution soft X-ray emission spectrometer attached to a EPMA clearly show a difference in intensity profile of $\mathrm{Fe}-\mathrm{L} \alpha$ emission of metal-Fe and $\mathrm{Fe} 2 \mathrm{O} 3$, and also show a presence of structure in $\mathrm{Ll}$ line due to the $3 \mathrm{~s} 3 \mathrm{~d}$ exchange interaction [7]. 


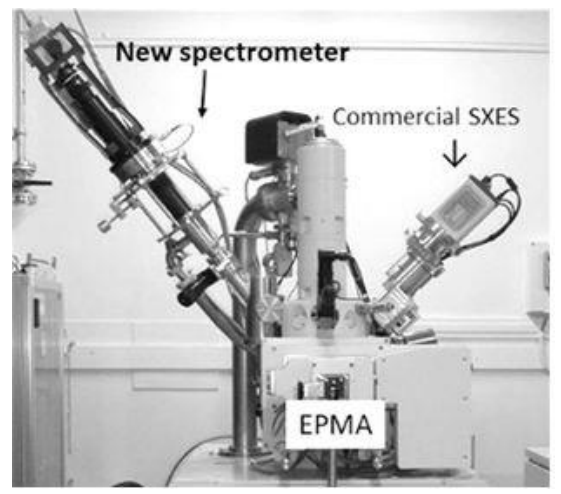

Figure 1. Photo of a newly constructed large-dispersion SXES spectrometer attached to EPMA.
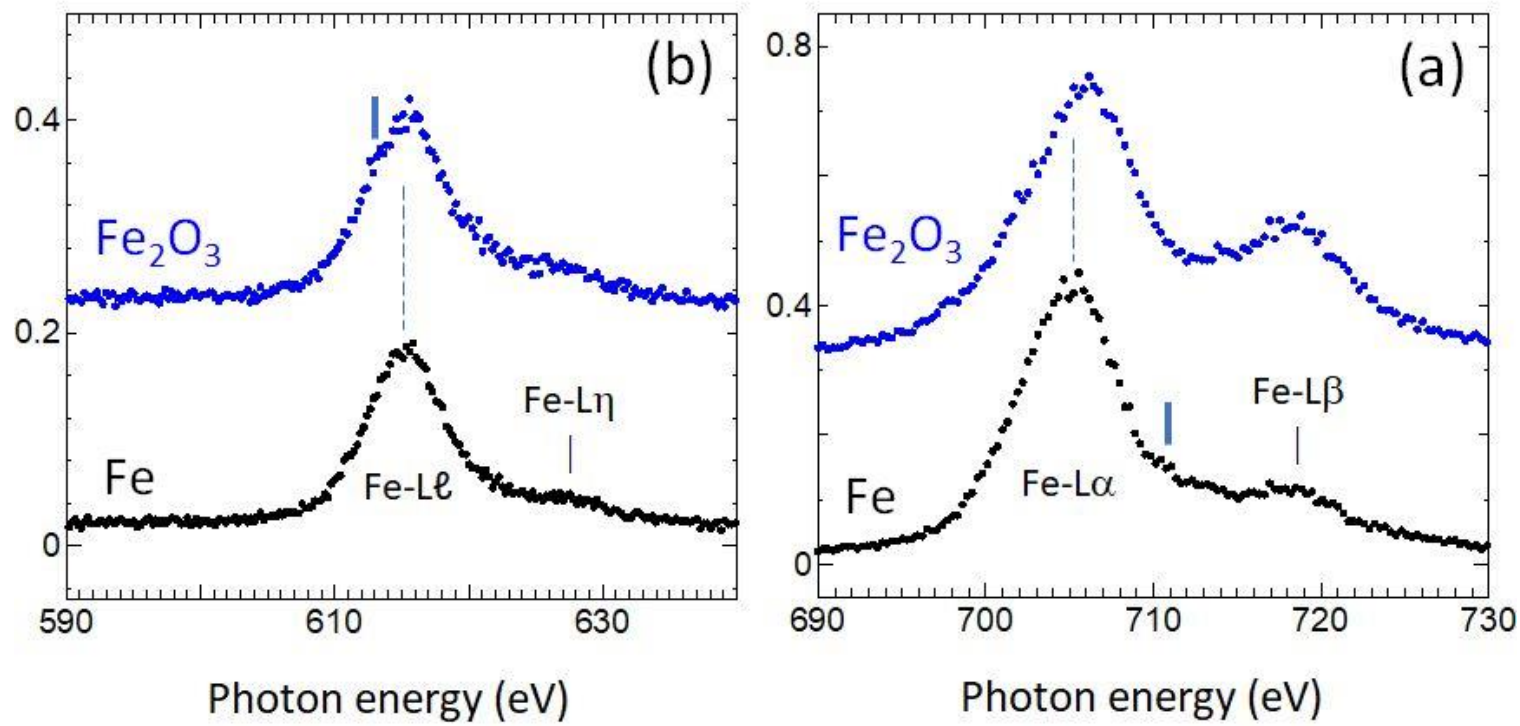

Figure 2. (a) Fe L $\alpha, \beta$-emission and (b) L $\ell, \eta$-emission spectra of meta-Fe and $\mathrm{Fe} 2 \mathrm{O} 3$.

\section{References}

1. Terauchi, M., Chap. 7 in "Transmission Electron Microscopy Characterization of Nanomaterials" (ed. Kumar C S S R, Springer-Verlag Berlin Heidelberg, 2014)

2. Terauchi, M., Microscopy 68, 330-337 (2019).

3. Terauchi, M., et al., IOP Conf. Series: Materials Science and Engineering 304, 012018 (2017).

4. Terauchi, M., et al., J. of Electron Microscopy 59, 251-261 (2010).

5. Terauchi, M. et al., IOP Conf. Series: Materials Science and Engineering 891, 012022 (2020).

6. Peng G., et al., J. Am. Chem. Soc. 116, 2914-2920 (1994).

7. This work was partly supported by the Research Program of "Dynamic Alliance for Open Innovation Bridging Human, Environment and Materials" in "Network Joint Research Centre for Materials and Devices" 